\title{
Oxidative Stability of Free Fatty Acid Mixtures from Soybean, Linseed, and Sardine Oils in an Aqueous Solution
}

\author{
Kazuo Miyashita, Naohiro Tateda, and Toru Ota \\ Faculty of Fisheries, Hokkaido University, Minato, Hakodate, Hokkaido 041, Japan \\ (Received November 12, 1993)
}

\begin{abstract}
The oxidative stability of free fatty acid (FFA) mixtures from soybean, linseed, and sardine oils in an aqueous solution was compared with that in air. Due to its high contents of DHA and EPA, sardine oil FFA was most rapidly oxidized in air, followed by linseed and soybean oil FFAs. However, this order was reversed in autoxidation in an aqueous solution $\left(\mathrm{pH}=7.4\right.$ at $\left.37^{\circ} \mathrm{C}\right)$. Gas chromatographic analysis showed that sardine oil FFA was most oxidatively stable in the aqueous solution and about $78 \%$ of polyunsaturated fatty acids (PUFAs) remained unchanged after $168 \mathrm{~h}$ of oxidation, while soybean oil FFA was highly susceptible to autoxidation in the aqueous solution, losing more than $80 \%$ of PUFAs after only $40 \mathrm{~h}$ of oxidation. This unusual order of oxidative stability in an aqueous solution can be explained by correlation with the micellar conformations of individual FFA mixtures in an aqueous solution.
\end{abstract}

Key words: oxidative stability, PUFA, DHA, EPA

Oxidative deterioration of unsaturated lipids is one of the most important problems in the field of food chemistry, because lipid oxidation products not only cause undesirable flavors but also decrease the nutritional quality and safety of lipid-containing foods. The oxidation rate of polyunsaturated fatty acids (PUFAs) is directly proportional to the number of bisallylic positions present in their molecules. $^{1-6)}$ Therefore, it is generally accepted that fish oils, containing high levels of n-3 PUFAs such as docosahexaenoic acid (DHA, 22:6n-3) and eicosapentaenoic acid (EPA, 20:5n-3), are highly susceptible to autoxidation. ${ }^{5,7)}$ However, these results have been mainly obtained by autoxidation of the purified substrate itself in air. In our previous paper, ${ }^{8)}$ we demonstrated that the oxidative stability of PUFA in an aqueous system is different from that in the neat phase and that DHA and EPA are oxidatively more stable than linoleic acid in an aqueous solution.

In the present study, we report the oxidative stability of sardine oil FFA, linseed oil FFA, and soybean oil FFA in an aqueous solution and compare it with that in the neat phase. The data described here will be useful for developing new approaches to stabilizing fish oils against autoxidation.

\section{Materials and Methods}

\footnotetext{
Materials

Soybean oil was purchased from Nacalai Tesque Inc., Kyoto Japan. Linseed oil was kindly donated from the Tsukuba Research Laboratory of Nippon Oil Fats Co., Tsukuba, Japan. Sardine oil was obtained from industrial plants in Hakodate, Japan. Each oil was fractionated by silicic acid column chromatography and the triacylglycerol eluted with etherhexane $(5: 95, v / v)$ was saponified, then the unsaponifiables such as tocopherols were removed with diethyl ether. The aqueous solution was acidified with $1 \mathrm{~N} \mathrm{HCl}$, and the FFA mixture was extracted with diethyl ether. The recovered mixture was refined just before processing by silicic acid column chromatography. The pure FFA sample gave only a single spot on the thin layer choromatogram and its peroxide value (PV) determined by the colorimetric iodine $\operatorname{method}^{9)}$ was zero. The fatty acid
}

composition of each FFA mixture was analyzed by capaillary gas chromatography (GC) after conversion to methyl esters by heating in a sealed tube at $90-100^{\circ} \mathrm{C}$ for $15 \mathrm{~min}$ with $7 \% \mathrm{BF}_{3}-\mathrm{MeOH}$. The capillary GC analysis was performed on a Shimadzu GC-14A (Shimadzu Seisakusho Co., Ltd., Kyoto, Japan) equipped with a flame-ionization detector and a fused-silica capillary column (Omegawax 320;30 $\mathrm{m} \times 0.32 \mathrm{~mm}$ i.d.; Supelco, Inc., Bellefonte, PA, U.S.A.). Hydrogen was used as a carrier gas and peak integration was carried out with a Shimadzu Chromatopac C-R6A integrator. The oven temperature was $195^{\circ} \mathrm{C}$, and the detector and injector temperatures were $230^{\circ} \mathrm{C}$.

Autoxidation of Soybean, Linseed, and Sardine Oil FFAs in Air

A $3 \mathrm{~g}$ sample of each FFA mixture in a flat-bottomed glass tube $(30 \mathrm{ml}$, $2.6 \mathrm{~cm}$ i.d.) was autoxidized by incubation in the dark at $37^{\circ} \mathrm{C}$. An aliquot of the sample was taken from the oxidized sample at certain time intervals to determine the changes in PV, total aldehyde content, and fatty acid composition.

The increase in total aldehyde content in oxidized FFA mixture was measured as described in our previous paper. ${ }^{10}$ ) The changes in the fatty acid composition during autoxidation of each FFA mixture were analyzed by fused-silica capillary GC after conversion of the FFAs to the corresponding methyl esters and silicic acid column chromatographic purification of these esters. The GC conditions were the same as those described above. The decrease in each PUFA by oxidation was estimated from the changes in its peak ratio to the inherent stearic acid.

Autoxidation of Soybean, Linseed, and Sardine Oil FFAs in an Aqueous Solution

Each purified FFA mixture $(50 \mathrm{mg}$ ) was dispersed in $100 \mathrm{~m} l$ of $0.05 \mathrm{M}$ phosphate buffer $\left(\mathrm{pH}=7.4\right.$ at $\left.37^{\circ} \mathrm{C}\right)$ containing $1 \%(w / v)$ of Tween 20 at $37^{\circ} \mathrm{C}$ in the dark. This substrate solution was transported into a $200 \mathrm{~m} l$ of erlenmeyer flask stoppered with a ground stopper and then incubated at $37^{\circ} \mathrm{C}$ in the dark.

After certain time intervals, $0.5 \mathrm{~m} l$ of oxidized solution was placed in a $10 \mathrm{~m} l$ calibrated flask and diluted to the mark with water. The UV absorption at $235 \mathrm{~nm}$ was measured with a Hitachi U-2000 spectrophotometer (Hitachi Seisakusho, Co., Tokyo, Japan). For GC analysis, $10 \mathrm{ml}$ of the solution was taken and oxidized FFAs were extracted twice with $\mathrm{CHCl}_{3}-\mathrm{MeOH}(2: 1, \mathrm{v} / \mathrm{v})$ after acidifing with $1 \mathrm{~N} \mathrm{HCl}$. The extract was washed with water, dried over anhydrous sodium sulfate, and concentrated in vacuo. The recovered FFAs were converted to methyl esters with $7 \%$ $\mathrm{BF}_{3}-\mathrm{MeOH}$ and then subjected to silicic acid column chromatography and to GC. The GC conditions and calculation of decrease in PUFA by oxidation were the same as those described above. These GC and UV 
measurements of the oxidative stability of FFA mixtures in an aqueous solution were carried out in duplicate at different intervals of sample collection. Since there was no significant differences in the results obtained from both experiments, the graphs of GC and UV analyses (Figs. 4, 5, and 6) are based on representative data from either of the duplicate results.

Prior to these experiments, small-scale aqueous oxidation with $10 \mathrm{~m} /$ of the substrate solution was carried out. The substrate solution in a flat-bottomed glass tube $\left(30 \mathrm{~m} l, 2.6 \mathrm{~cm}\right.$ i.d.) was incubated at $37^{\circ} \mathrm{C}$ in the dark for $24 \mathrm{~h}$, and the oxidative stability of each FFA mixture was analyzed by $\mathrm{GC}$ as described above. This preliminary experiment was done in triplicate.

\section{Results and Discussion}

The fatty acid compositions of FFA mixtures from soybean, linseed, and sardine oil are summarized in Table 1. The major PUFAs in the sardine oil FFA were docosahexaenoic acid (22:6n-3, DHA) and eicosapentaenoic acid (20:5n-3, EPA). On the other hand, linseed and soybean oil FFAs contained more than $50 \%$ of $\alpha$-linolenic acid (18:3n-3, $\alpha$-LNA) and linoleic acid (18:2n-6, LA), respectively.

These characteristic PUFA compositions of each FFA mixture strongly influenced its oxidative stability (Figs. 1 and 3). The changes in the total amounts of PUFAs during autoxidation of the FFA mixture (Fig. 1) show that sardine oil FFA was most easily oxidized in air, followed by linseed

Table 1. Composition of main fatty acids of soybean, linseed, and sardine oil FFAs

\begin{tabular}{lccr}
\hline FFA & Soybean & Linseed & Sardine \\
\hline $14: 0$ & 0.07 & 0.05 & 8.65 \\
$16: 0$ & 11.30 & 4.97 & 20.10 \\
$18: 0$ & 3.69 & 2.71 & 2.41 \\
$16: 1 n-7$ & 0.15 & - & 6.63 \\
$18: 1 n-7$ & 1.64 & 0.65 & 3.05 \\
$18: 1 n-9$ & 19.89 & 15.80 & 9.31 \\
$20: 1 n-9$ & 0.14 & - & 1.74 \\
$20: 1 \mathrm{n}-11$ & - & - & 2.79 \\
$22: 1 \mathrm{n}-11$ & - & - & 3.03 \\
$18: 2 \mathrm{n}-6$ & 56.11 & 14.27 & 1.79 \\
$18: 3 \mathrm{n}-3$ & 6.03 & 60.51 & 1.15 \\
$18: 4 \mathrm{n}-3$ & - & - & 3.09 \\
$20: 5 \mathrm{n}-3$ & - & - & 11.20 \\
$22: 5 \mathrm{n}-3$ & - & - & 1.65 \\
$22: 6 \mathrm{n}-3$ & - & - & 11.60 \\
\hline
\end{tabular}

oil and soybean oil FFAs.

Figure 2 shows the decrease in the amount of individual unsaturated fatty acid (UFA) classes during autoxidation of three kinds of FFA mixture. In all three cases, the oxidation rate of the unoxidized fatty acid classes in the mixture increased with increasing degree of unsaturation.

The high susceptibility of sardine oil FFA to autoxidation in air was also confirmed by measuring the PV and total aldehyde content (Fig. 3). A rapid increase in these values during autoxidation of sardine oil FFA was observed without any induction period of autoxidation. However, in the case of soybean oil FFA, they increased after a long induction period.

In contrast to autoxidation in air, sardine oil FFA was most oxidatively stable in an aqueous solution, followed by linseed oil FFA and soybean oil FFA (Figs. 4 and 5). When sardine oil FFA was oxidized in an aqueous solution at $37^{\circ} \mathrm{C}$ in the dark, about $15 \%$ of PUFAs was oxidized by incubation for $24 \mathrm{~h}$, but after that, only a slight decrease in the PUFA content was observed and more than $78 \%$ of PUFAs remained unchanged after $168 \mathrm{~h}$ of oxidation (Fig. 5). In contrast, linseed oil FFA and soybean oil FFA were oxidized with a loss of about $80 \%$ of PUFAs after $40 \mathrm{~h}$ and $96 \mathrm{~h}$ of oxidation, respectively. Furthermore, this characteristic oxidative stability of FFA mixtures in an aqueous solution was also confirmed by the small-scale preliminary experiments, which indicated that the percentage loss of PUFAs after $24 \mathrm{~h}$ of oxidation of sardine oil FFA, linseed

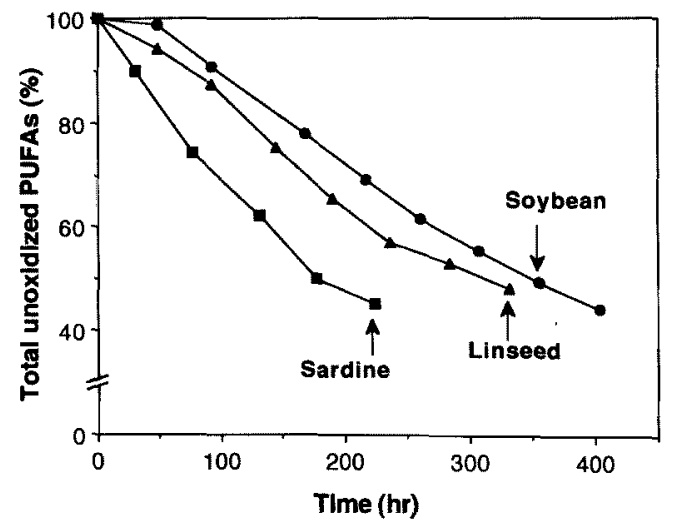

Fig. 1. Changes in the total amount of PUFAs during autoxidation of soybean, linseed, and sardine oil FFAs in air at $37^{\circ} \mathrm{C}$ in the dark.
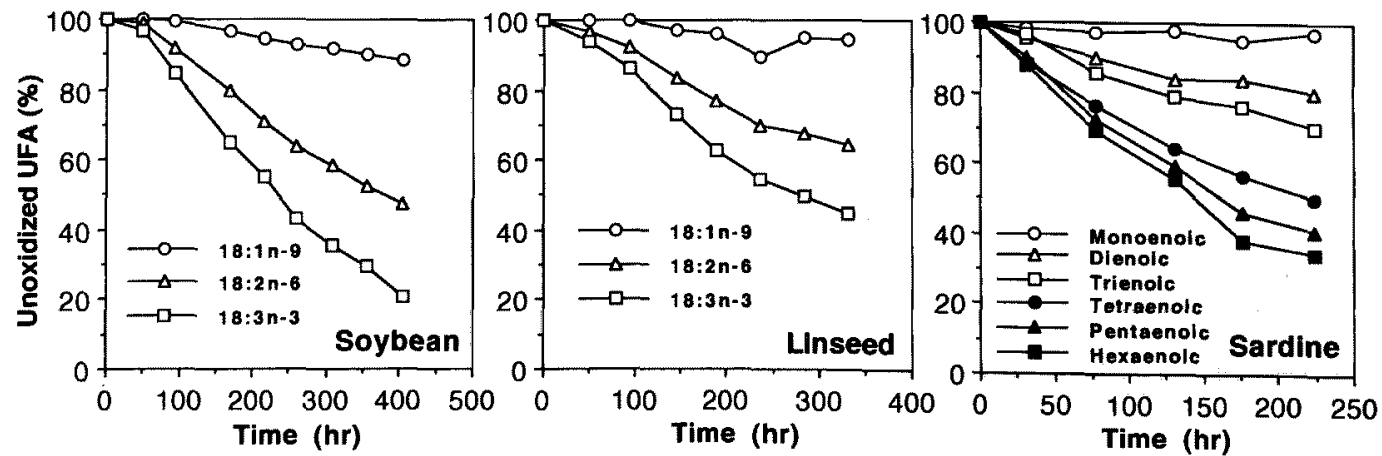

Fig. 2. Changes in the amount of individual UFA classes during autoxidation of soybean, linseed, and sardine oil FFAs in air at $37^{\circ} \mathrm{C}$ in the dark. 

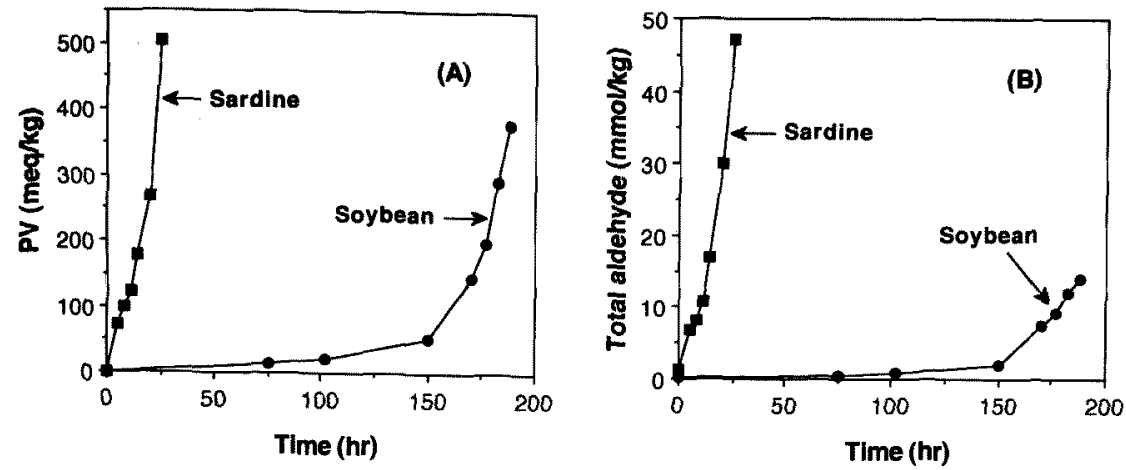

Fig. 3. Changes in PVs (A) and total aldehyde content (B) during autoxidation of soybean and sardine oil FFAs in air at $37^{\circ} \mathrm{C}$ in the dark.

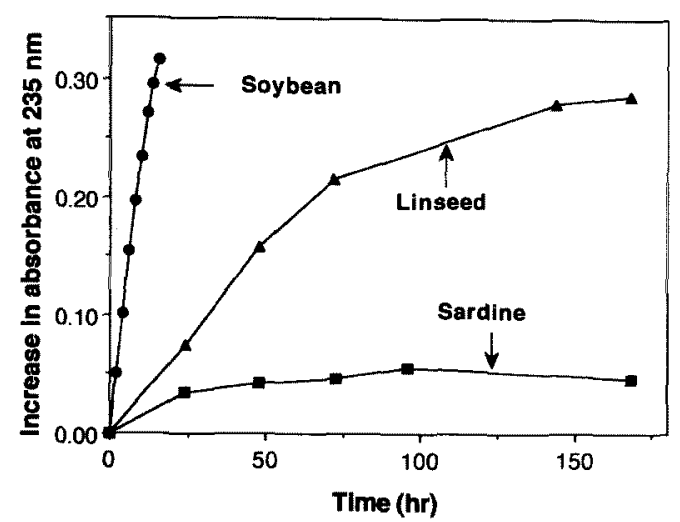

Fig. 4. Increases in UV absorption at $235 \mathrm{~nm}$ during autoxidation of soybean, linseed, and sardine oil FFAs in an aqueous solution at $37^{\circ} \mathrm{C}$ in the dark.

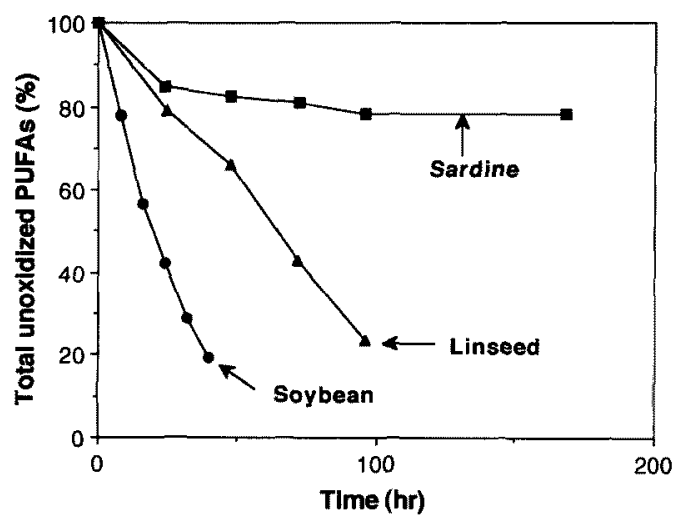

Fig. 5. Changes in the total amount of PUFAs during autoxidation of soybean, linseed, and sardine oil FFAs in an aqueous solution at $37^{\circ} \mathrm{C}$ in the dark.
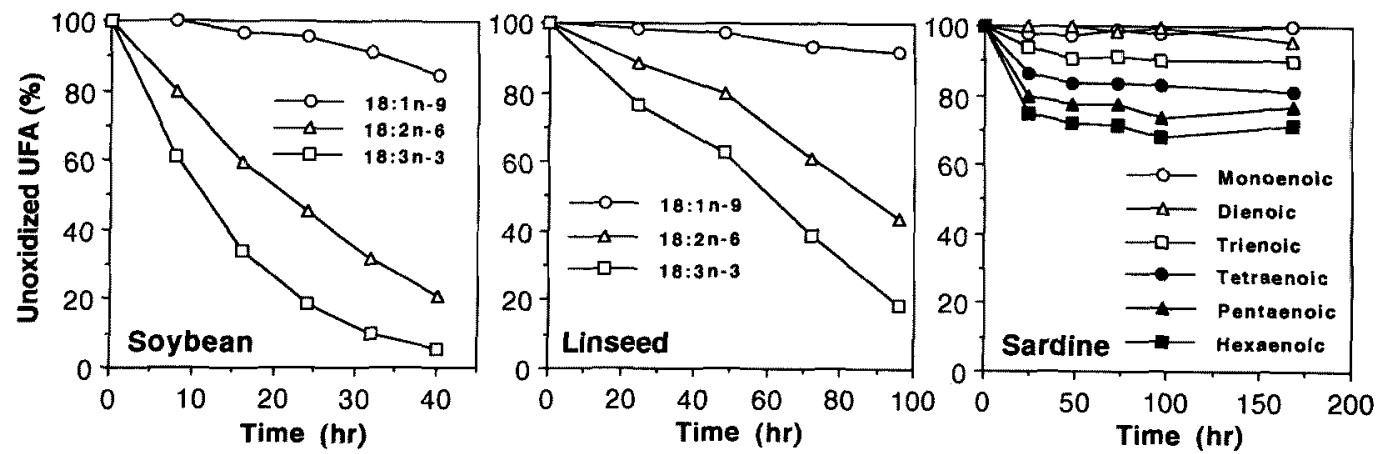

Fig. 6. Changes in the amount of individual UFA classes during autoxidation of soybean, linseed, and sardine oil FFAs in an aqueous solution at $37^{\circ} \mathrm{C}$ in the dark.

oil FFA, and soybean oil FFA were $11 \pm 1.2,22 \pm 3.3$, and $55 \pm 1.9$ (mean \pm S.D.), respectively.

The unusual order of oxidative stability of FFA mixtures in the aqueous solution can be explained by correlation with the micellar conformations in the aqueous medium. The micelles composed of sardine oil FFA can form a more compact and tighter conformation than those of the other FFA mixtures and this conformation may have increased the stability of the micelles against attack by free radicals or oxygen. The higher oxidative stability of linseed oil FFA than soybean oil FFA may be due to the difference in the micellar conformations of both FFAs. Similar observa- tions have been made in the oxidation of polyunsaturated phosphatidylcholine (PC) by lipoxygenase. ${ }^{11)}$ The reactivity of $\mathrm{PC}$ was dependent on the micellar nature of the $\mathrm{PC}$ in an aqueous solution.

As shown in Table 1, sardine oil FFA and linseed oil FFA were characterized by their high content of long-chain n-3 PUFAs such as DHA, EPA, and $\alpha$-LNA. Therefore, it is suggested that the oxidative stability of FFA micelles increases with increasing concentration of n-3 PUFA content, especially the DHA and EPA content. The same observation was made in our previous oxidation of DHA/ LA mixtures with $\mathrm{Fe}$ (II)-ascorbic acid (AsA) as a radical 
initiator. ${ }^{8)}$ The oxidative stability of the DHA/LA mixture increased with increasing molar ratio of DHA to LA. It is suggested that the nature of the radical initiator is strongly correlated with the oxidative stability of PUFA in a micellar phase. ${ }^{12)}$ The different oxidative stabilities of PUFA from the present and the previous studies may be obtained when an oil-soluble radical initiator such as 2,2'-azobis(2,4-dimethylpentanenitrile) was used for aqueous oxidation of PUFA.

Figure 6 shows the decreases in the amount of unoxidized UFA classes during oxidation of FFA mixtures in an aqueous solution. The order of decrease of each class of the unoxidized fatty acid is the same as that found in autoxidation in air (Fig. 2), although the oxidative stability of FFA mixtures in an aqueous solution was quite different from that in air. These results suggest that FFA micelles form a more oxidatively stable conformation with increasing concentration of n-3 PUFAs such as DHA, EPA, and $\alpha$ LNA, but that once the FFA micelle is oxidized, the more highly PUFAs are more rapidly oxidized.

Acknowledgments This work was supported in part by a Grant-in-Aid for Scientific Research (No. 05660225) from the Ministry of Education, Science, and Culture of Japan.

\section{References}

1) F. D. Gunstone and T. P. Hilditch: The union of gaseous oxygen with methyl oleate, linoleate, and linolenate. J. Chem. Soc., 836-841
(1945).

2) R. T. Holman and O.C. Elmer: The rates of oxidation of unsaturated fatty acids and esters. J. Am. Oil Chem. Soc., 24, 127-129 (1947).

3) K. Miyashita and T. Takagi: Study on the oxidative rate and prooxidant activity of free fatty acids. $J$. Am. Oil Chem. Soc., 63, 1380-1384 (1986).

4) J. P. Cosgrove, D. F. Church, and W. A. Pryor: The kinetics of the autoxidation of polyunsaturated fatty acids. Lipids, 22, 299-304 (1987).

5) S.-Y. Cho, K. Miyashita, T. Miyazawa, K. Fujimoto, and T. Kaneda: Autoxidation of ethyl eicosapentaenoate and docosahexaenoate. $J$. Am. Oil Chem. Soc., 64, 876-879 (1987).

6) K. Miyashita, E. N. Frankel, W. E. Neff, and R. A. Awl: Autoxidation of polyunsaturated triacylglycerols. III. synthetic triacylglycerols containing linoleate and linolenate. Lipids, 25, 48-53 (1990).

7) K. L. Fritsche and P. V. Johnston: Rapid autoxidation of fish oil in diets without added antioxidants. J. Nutr., 118, 425-426 (1988).

8) K. Miyashita, E. Nara, and T. Ota: Oxidative stability of polyunsaturated fatty acids in an aqueous solution. Biosci. Biotech. Biochem., 57, 1638-1640 (1993).

9) T. Takagi, Y. Mitsuno, and M. Masumura: Determination of peroxide value by the colorimetric iodine method with protection of iodide as cadmium complex. Lipids, 13, 147-151 (1978).

10) K. Miyashita, K. Kanda, and T. Takagi: A simple and quick determination of aldehydes in autoxidized vegetable and fish oils. $J$. Am. Oil Chem. Soc., 68, 748-751 (1991).

11) J. Eskola and S. Laakso: Bile Salt-dependent oxygenation of polyunsaturated phosphatidylcholines by soybean lipoxygenase-1. Biochim. Biophys. Acta, 751, 305-311 (1983).

12) Y. Yamamoto, S. Haga, E. Niki, and Y. Kamiya: Oxidation of Lipids. V. Oxidation of methyl linoleate in aqueous dispersion. Bull. Chem. Soc. Jpn., 57, 1260-1264 (1984). 